\title{
Propofol-based deep sedation for percutaneous radiofrequency ablation in sick elderly patients with hepatocellular carcinoma in a developing country
}

\begin{abstract}
The aim of this study was to evaluate and compare the clinical efficacy of propofol-based deep sedation (PBDS) for percutaneous radiofrequency ablation (RFA) in sick (American Society of Anesthesiologists [ASA] physical status III-IV) and nonsick (ASA physical status I-II) elderly patients in a teaching hospital in Thailand. We undertook a retrospective review of the sedation service records of elderly patients who underwent RFA procedures from 2010 to 2012. Patients were classified into two groups according to ASA physical status. In group A, patients had ASA I-II, while in group B, patients had ASA III-IV. Primary outcome variable was the successful completion of procedure. Secondary outcome variables were sedation-related complications during and immediately after procedure. There were 113 elderly patients who underwent RFA procedures by using PBDS during the study period. Of these, 69 patients were in group A and 44 patients were in group B. There were no significant differences in age, weight, duration of sedation, and mean dose of propofol, fentanyl, and midazolam between the two groups. All procedures in both groups were successfully completed. Overall and cardiorespiratory-related complications in both groups were not significantly different. All sedation-related complications were easily treated, with no adverse sequelae. PBDS for percutaneous RFA in sick elderly patients by trained anesthetic personnel with appropriate monitoring was safe and effective. The clinical efficacy of this technique in sick elderly patients was not different or worse than in nonsick elderly patients. Serious complications were rare.
\end{abstract}

Keywords: deep sedation, propofol, percutaneous radiofrequency ablation, hepatocellular carcinoma, elderly, sick, developing country
Volume 8 Issue 3 - 2017

Somchai Amornyotin, Siriporn Kongphlay

Department of Anesthesiology and Siriraj GI Endoscopy Center, Faculty of Medicine Siriraj Hospital, Mahidol University, Thailand

Correspondence: Somchai Amornyotin, Department of Anesthesiology and Siriraj GI Endoscopy Center, Faculty of Medicine Siriraj Hospital, Mahidol University, 2, Siriraj, Bangkoknoi, Bangkok 10700, Thailand, Tel +662 419 7990, Fax +6624II 3256, Email somchai.amo@mahidol.ac.th

Received: May II, 2017 | Published: July 06, 2017
Abbreviations: PBDS, propofol-based deep sedation; RFA, radiofrequency ablation; ASA, American Society of Anesthesiologists; $\mathrm{SD}$, standard deviation; \%, percentage

\section{Introduction}

Radiofrequency ablation (RFA) is one of the most common interventional medical procedures performed throughout the world. It is an alternative treatment option for hepatic tumor patients. These tumors are ablated by radiofrequency. Radiofrequency is the heating and destruction of tissues in which a high-frequency alternating current increases the temperature of the tissues beyond $60^{\circ} \mathrm{C}$, causing in a region of necrosis nearby the electrode. This ultrasonic energy is a strong stimulus that requires deep sedation and anesthesia levels during the radiofrequency procedure. RFA is considered to be equally effective as surgical resection in the patients with solitary tumor nodules of $\leq 2 \mathrm{~cm} .{ }^{1}$ However, the appropriate method of treatment still depends on several critical factors including age, patient physical status, and severity of hepatic dysfunction.

Over the years, the patients also have changed. There are increasingly patients at the extremes of age and health status as well as patients with a multitude of comorbid problems. In our center, most of these procedures are performed by radiologists under some forms of anesthesia. The choices and techniques of anesthesia and drug selection vary according to the condition of the patients, familiarity of the anesthesiologists and satisfaction of the radiologists. ${ }^{2}$ Little is known about how practices in anesthesia during RFA procedure in the radiology unit outside the operating room in the developing countries. Consequently, the anesthesia-related complication rates have changed over time. The aim of this study was to evaluate and compare the clinical efficacy of propofol-based deep sedation (PBDS) for percutaneous RFA procedure in sick (American Society of Anesthesiologists [ASA] physical status III-IV) and nonsick (ASA physical status I-II) elderly patients with hepatocellular carcinoma in a teaching hospital in Thailand.

\section{Materials and method}

\section{Patients}

The patients from Siriraj Hospital, Bangkok, Thailand underwent percutaneous RFA procedures from 2010 to 2012 were eligible for the study. Inclusion criteria were age $>65$ years, and percutaneous RFA procedures performed using PBDS technique. Exclusion criteria were the RFA procedures performed in the intensive care units, procedures performed without PBDS, or procedures performed under monitored anesthesia care and general anesthesia.

\section{Study design}

This study is a retrospective descriptive study. All elderly patients were classified into two groups according to the ASA physical status. In group A, patients had ASA I-II. In group B, patients had ASA IIIIV. The primary outcome variable of the study was the successful completion of procedure. Secondary outcome variables were sedationrelated adverse events during and immediately after procedure. Successful percutaneous RFA procedure was defined as completion of the procedure as intended. Failed procedure is defined as the procedure 
which cannot be completed by using PBDS technique or the sedationrelated serious adverse events such as severe hypoxemia $\left(\mathrm{SpO}_{2}<85 \%\right.$ more than 3 minutes and cannot relief by airway management), severe cardiorespiratory instability.

\section{Sedation-related complications}

All serious and minor complications were recorded. Serious complication was defined as severe hypoxia (oxygen saturation $\left(\mathrm{SpO}_{2}\right)<85 \%,>3$ minutes). Minor complications were defined as follows: transient desaturation $\left(\mathrm{SpO}_{2}<90 \%\right)$; airway obstruction; hypertension or hypotension (increase or decrease in blood pressure by $25 \%$ from baseline); tachycardia or bradycardia (increase or decrease in heart rate by $25 \%$ from baseline); any cardiac arrhythmias. Airway intervention was defined as airway management (head tilt, chin lift, jaw thrust) or utilization of airway device (oropharyngeal or nasopharyngeal airway). After the procedure, all patients were observed in the recovery room at least two hours before discharged to ward.

\section{Statistical analysis}

Results were expressed as mean \pm SD or percentage (\%), when appropriate. Comparisons between group A and B were compared by using with Chi-square tests (for categorical variables), Chi-square tests for trend (for ordinal variables), and two-sample independent $t$-test (for continuous variables). The statistical software package SPSS for Window Version 18 (SPSS Inc., Chicago, IL) was used to analyze the data. All statistical comparisons were made at the two-sided 5\% level of significance.

\section{Results}

There were 113 elderly patients who underwent percutaneous RFA procedures by using PBDS during the study period. Of these, 69 patients were in group A and 44 patients were in group B. There were no significant differences in age, weight, duration of sedation, and mean dose of propofol, fentanyl, and midazolam between the two groups. All procedures in both groups were successfully completed. Overall and cardiorespiratory-related complications in both groups were not significantly different. All sedation related complications were easily treated, with no adverse sequelae.

Table 1 showed the characteristics of patients, duration of sedation and indications of procedure. There were no statistically significant differences in age, weight, height, body mass index, sedation time and indication of the procedure between the two groups. However, the female gender in group A was significantly greater than in group B.

Cardiovascular monitoring, including noninvasive blood pressure measurements, electrocardiogram, heart rate, and oxygen saturation, was performed. No pre-medications were used before the procedure. All patients were sedated by well-trained anesthetic personnel directly supervised by a staff anesthesiologist in the endoscopy room. Anesthetic personnel included residents in anesthesiology and anesthetic nurses who were well trained in the use of sedation technique and airway management. All patients were sedated in deep sedation level, according to guideline of the American Society of Anesthesiologists. ${ }^{3}$ Subsequently, all cases were concluded with the satisfactory completion of the procedure.

Table 2 demonstrated successful completion rate of procedure, overall complication rate and sedation-related complications. All procedures were successfully completed. There were no significant differences in overall complication rate and sedation related complications between the two groups. Airway interventions were not significantly different between the two groups. No procedures were aborted as a result of insufficient sedation or complications of sedation.

Hemodynamic parameters including systolic and diastolic blood pressure, heart rate and oxygen saturation were demonstrated in Table 3. There were not significant differences between the groups in hemodynamic parameters at baseline, start ablation and at the other time periods after ablation. Oxygen saturation in both groups was over $99 \%$ throughout the study period.

Table I Characteristics of patients, duration of sedation and indication of procedure (Mean, SD and \%)

\begin{tabular}{llll}
\hline & Group A (n=69) & Group B (n=44) & P Value \\
\hline Age (yr) (mean, SD) & $72.7(5.4)$ & $74.3(5.6)$ & 0.5 \\
Gender (\%): & & & $0.018^{*}$ \\
Male & $40(58.0)$ & $35(79.5)$ & \\
Female & $29(42.0)$ & $9(20.5)$ & \\
Weight (kg) (mean, SD) & $62.2(10.6)$ & $65.3(11.1)$ & 0.672 \\
Height (cm) (mean, SD) & $161.2(8.2)$ & $162.3(7.9)$ & 0.486 \\
Duration of sedation (min) (mean, SD) & $31.8(14.8)$ & $90.3(54.0)$ & 0.934 \\
\hline
\end{tabular}

Group A, ASA physical status I, II; Group B, ASA physical status III, IV

*Considered statistically significant

Table 2 Successful completion of procedure, overall complication rate and sedation-related complications ( $\mathrm{n}, \%)$

\begin{tabular}{llll}
\hline & Group A (n=69) & Group B (n=44) & P Value \\
\hline $\begin{array}{l}\text { Successful completion of procedure } \\
\text { Overall complication rate }\end{array}$ & $69(100.0)$ & $44(100.0)$ & $\mathrm{I}$ \\
$\begin{array}{l}\text { Sedation-related complications } \\
\text { Hypotension }\end{array}$ & $16(23.2)$ & $21(47.7)$ & 0.276 \\
$\begin{array}{l}\text { Hypertension } \\
\text { Bradycardia }\end{array}$ & $1 \mathrm{I}(15.9)$ & $16(36.4)$ & \\
\hline
\end{tabular}

Group A,ASA physical status I, II; Group B,ASA physical status III, IV 
Table 3 Hemodynamic parameters: systolic and diastolic blood pressure $(\mathrm{mmHg})$, heart rate (beat/minute) and oxygen saturation (SpO ${ }_{2}$ \%) (Mean, $\left.\mathrm{SD}\right)$

\begin{tabular}{|c|c|c|c|}
\hline & Group A (n=69) & Group $B(n=44)$ & PValue \\
\hline \multicolumn{4}{|l|}{ Baseline } \\
\hline SBP, DBP & | 44.5 (19.1), 79.4 (12.4) & | 40.1 (25.0), 76.7 (I2.9) & $0.496,0.558$ \\
\hline $\mathrm{HR}, \mathrm{SpO}_{2}$ & 68.4 (II.6), $99.9(0.2)$ & $68.2(12.3), 99.9(0.2)$ & $0.542,0.150$ \\
\hline \multicolumn{4}{|c|}{ At start ablation } \\
\hline SBP, DBP & I 37.9 (20.4), 74.9 (| | .3) & I 34.4 (25.6), 73.7 (I3.0) & $0.617,0.121$ \\
\hline $\mathrm{HR}, \mathrm{SpO}_{2}$ & 67.5 (II.3), $100.0(0.1)$ & $68.6(14.0), 99.9(0.2)$ & $0.421,0.746$ \\
\hline \multicolumn{4}{|c|}{5 min after ablation } \\
\hline SBP, DBP & |35.| (22.2), 74.5 (12.6) & I 29.6 (23.5), $70.6($ II.I) & $0.398,0.513$ \\
\hline $\mathrm{HR}, \mathrm{SpO}_{2}$ & $67.4(12.1), 100.0(0.1)$ & $66.8(13.9), 100.0(0.0)$ & $0.083,0.422$ \\
\hline \multicolumn{4}{|c|}{10 min after ablation } \\
\hline SBP, DBP & I 32.7 (20.5), 73.6 (| I.2) & I $27.2(22.9), 69.8(12.1)$ & $0.510,0.777$ \\
\hline $\mathrm{HR}, \mathrm{SpO}_{2}$ & 68.0 (II.7), $100.0(0.0)$ & 66.9 (I3.3), I00.0 (0.0) & $0.214,1.000$ \\
\hline \multicolumn{4}{|c|}{ I5 min after ablation } \\
\hline SBP, DBP & I 32.0 (23.5), 73.9 (13.3) & I $26.6(22.3), 69.2(11.2)$ & $0.373,0.833$ \\
\hline $\mathrm{HR}, \mathrm{SpO}_{2}$ & 67.6 (12.3), $100.0(0.0)$ & 66.4 (I2.9), I00.0 (0.0) & $0.564,1.000$ \\
\hline \multicolumn{4}{|c|}{20 min after ablation } \\
\hline SBP, DBP & I 29.8 (23.6), 72.1 (13.2) & I $24.6(24.4), 68.3(13.8)$ & $0.394,0.697$ \\
\hline $\mathrm{HR}, \mathrm{SpO}_{2}$ & $67.5(12.1), 100.0(0.0)$ & $66.0(14.6), 100.0(0.0)$ & $0.377,1.000$ \\
\hline \multicolumn{4}{|c|}{25 min after ablation } \\
\hline SBP, DBP & I 28.9 (22.9), 73.2 (13.7) & I $24.8(27.6), 67.8(15.6)$ & $0.342,0.774$ \\
\hline $\mathrm{HR}, \mathrm{SpO}_{2}$ & 68.6 (12.0), $100.0(0.0)$ & 67.9 (14.4), $99.9(0.2)$ & $0.339,0.192$ \\
\hline \multicolumn{4}{|c|}{30 min after ablation } \\
\hline SBP, DBP & I25.8 (2I.9), 71.6 (I5.2) & I $24.8(26.8), 68.5(\mid 4.2)$ & $0.503,0.764$ \\
\hline $\mathrm{HR}, \mathrm{SpO}_{2}$ & 68.1 (12.4), $100.0(0.0)$ & $68.7(14.3), 99.9(0.2)$ & $0.137,0.218$ \\
\hline \multicolumn{4}{|c|}{35 min after ablation } \\
\hline SBP, DBP & I 28.4 (2I.7), 73.2 (I4.0) & I 24.4 (23.4), 69.1 (I2.4) & $0.681,0.538$ \\
\hline $\mathrm{HR}, \mathrm{SpO}_{2}$ & 68.1 (12.4), $100.0(0.0)$ & $68.4(14.0), 99.9(0.2)$ & $0.621,0.231$ \\
\hline \multicolumn{4}{|c|}{40 min after ablation } \\
\hline SBP, DBP & I 32.4 (23.5), 74.6 (I5.I) & I 26.4 (2I.7), 70.0 (| 3.4$)$ & $0.677,0.358$ \\
\hline $\mathrm{HR}, \mathrm{SpO}_{2}$ & 68.2 (II.9), $100.0(0.0)$ & $69.0(14.2), 99.9(0.2)$ & $0.904,0.214$ \\
\hline \multicolumn{4}{|c|}{45 min after ablation } \\
\hline SBP, DBP & I 32.9 (22.5), 74.6 (13.5) & I $23.8(23.2), 68.6(\mid 4.9)$ & $0.461,0.307$ \\
\hline $\mathrm{HR}, \mathrm{SpO}_{2}$ & 70.0 (I2.0), $100.0(0.0)$ & $69.6(14.3), 99.9(0.2)$ & $0.804,0.230$ \\
\hline \multicolumn{4}{|c|}{50 min after ablation } \\
\hline SBP, DBP & | 3 I.4 (24.I), $74 . \mid$ (|3.8) & I $24.8(23.6), 68.6(14.9)$ & $0.535,0.489$ \\
\hline $\mathrm{HR}, \mathrm{SpO}_{2}$ & 70.7 (II.4), $100.0(0.0)$ & $70.2(15.5), 99.9(0.2)$ & $0.544,0.243$ \\
\hline \multicolumn{4}{|c|}{55 min after ablation } \\
\hline SBP, DBP & | 3 |.2 (26.2), 74.0 (|4.8) & I $26.8(26.5), 69.5(16.2)$ & $0.758,0.594$ \\
\hline $\mathrm{HR}, \mathrm{SpO}_{2}$ & 70.1 (II.3), $100.0(0.0)$ & $69.3(15.7), 99.9(0.2)$ & $0.288,0.27 \mid$ \\
\hline \multicolumn{4}{|c|}{60 min after ablation } \\
\hline SBP, DBP & |30.| (24.0), 73.6 (I3.0) & $122.8(23.3), 67.4(11.9)$ & $1.000,0.67 \mid$ \\
\hline $\mathrm{HR}, \mathrm{SpO}_{2}$ & $70.0(10.3), 100.0(0.0)$ & $68.6(16.4), 99.9(0.2)$ & $0.461,0.276$ \\
\hline
\end{tabular}

Group A, ASA physical status I, II; Group B, ASA physical status III, IV; SBP, systolic blood pressure; DBP, diastolic blood pressure; HR, heart rate; SpO, oxygen saturation

\section{Discussion}

RFA is a relatively new technique for the treatment of small hepatic tumors that cannot be treated with surgical procedure. This technique applies alternating high-frequency electrical currents to the cancerous tissue. The intense heat leads to thermal coagulation that can destroy the tumors. The technology of RFA has been enhanced over the past 20 years and the methods of anesthesia have been improved as well. ${ }^{4}$ However, RFA is an invasive procedure and is normally denied by the patient because of anxiety and severe discomfort and pain. Importantly, sedation and anesthesia can decrease the fear of the procedure by inducing amnesia and reducing pain and discomfort so that the patients can undergo the procedure in a comfortable state.

The role of the anesthesiologist in hepatocellular carcinoma ablation therapy is to facilitate patient safety and satisfaction as well as to ensure that the patient will have minimal pain during the procedure. To date, there is evidence that percutaneous RFA procedure can be safely performed with sedation. The PBDS technique is commonly used for this procedure. ${ }^{5}$ Although the use of sedation during this procedure is supposed to be extensively accepted, data from different studies indicate that patterns of use of sedative and analgesic drugs may principally depend on cultural, or even regional and local differences. However, the data used to evaluate the safety of PBDS during RFA procedure in sick elderly patients were derived mainly from several studies conducted in the developed countries. There are limited data in the developing countries.

Our study demonstrated that PBDS for percutaneous RFA procedure in sick elderly patients by trained anesthetic personnel with appropriate monitoring was safe and effective. The clinical efficacy of this technique in sick elderly patients was not different or worse than in nonsick elderly patients. The rate of sedation-related complication during this procedure was comparable. In addition, PBDS for sick elderly patients does not increase the overall complication rate in comparison to the nonsick elderly patients. However, the complication 
rate in this present study is relatively high. One possible explanation of this finding is that the number of RFA procedure underwent PBDS technique has remarkably increased over the last few years. The depth of sedation in our report was moderate to deep level. In addition, the complication rate would be correlated to the depth of sedation, directly. A previous study showed that dexmedetomidine provided better respiratory stability and reduced opioid consumption in comparison with propofol when administered under monitored anesthesia care (MAC) when performing RFA for hepatic neoplasm. For safer sadation of patients, we recommended dexmedetomidine when used in MAC during RFA for hepatic neoplasm than propofol. ${ }^{6}$

The incidence rates of complications per treatment and per procedure were $2.2 \%$ and $1.5 \%$, respectively. The treatment mortality rate was $0.03 \% .^{7}$ Complications of RFA can arise in three general ways. The first way develops from the ablative procedure itself. The second way results in complications relate to the RFA device passed through tissue to a target organ such as bleeding, pneumothorax, and infection. The third way associates with sedation and anesthesia. ${ }^{8}$ This study does not focus on procedure-related complications. In Siriraj Hospital, there were no serious sedation-related complications occurred during the study. Several previous studies also demonstrated that PBDS for various endoscopic procedures in elderly patients by trained anesthesia personnel with appropriate monitoring was relatively safe and effective. Although adverse cardiovascular events, including hypotension, in this aged group is common, all adverse events were usually transient, mild, and easily treated, with no sequelae..$^{9-11}$

Patients were breathing spontaneously; however, oxygen saturation was always over $99 \%$, and age, ASA physical status and the combination of sedative agents did not negatively influence this parameter. Sedation is performed to ensure the patient's safety, to minimize physical discomfort or pain, to provide analgesia and procedural amnesia, to control behavior during the procedure and to return the patient to pretreatment level of consciousness. The amount of sedation required depends on the patient's physical status and age. Propofol is widely employed for anesthesia outside the OR because it is easy to use, has a good safety and efficacy profile due to its quick onset of action, rapid metabolism, significantly shorter recovery time and it has some anti-emetic effects. ${ }^{12-14}$

Limitations of this study exist. First, the present report is retrospective in nature. The main limitation of this study is its reliance on self-reported data. The inaccurate and incomplete documentation of certain measures, as occurs with many chart reviews, also occurred in this study. These self-reporting data may tend toward an underestimation of unpleasant data. Second, this is a single-center study. These results could not be reproducible constantly in other settings. Third, the limitation of monitoring such as of end-tidal carbondioxide, could result in a lower rate of adverse events. Overall, despite these limitations, we are, however, confident that these findings are generalizable to the practice of percutaneous RFA procedure using PBDS technique. Finally, because the serious complications in our series were low, further studies in larger prospective groups of patients are therefore needed.

\section{Conclusion}

The authors report the performance of the clinical efficacy of PBDS regimen utilizing anesthesiologist or anesthetic personnel with appropriate basic monitoring for percutaneous RFA procedure in a tertiary-care teaching hospital in Thailand. The findings of the present study demonstrated that PBDS for this procedure in sick elderly patients by trained anesthetic personnel with appropriate monitoring was safe and effective. The clinical efficacy of this technique in sick elderly patients was not different or worse than in nonsick elderly patients. Serious complications were rare. We hope that our practice will help model the development of PBDS for percutaneous RFA procedure in the developing countries.

\section{Acknowledgments}

None.

\section{Conflicts of interest}

The authors declare that they have no competing interests.

\section{References}

1. Livraghi T, Meloni F, Di Stasi M, et al. Sustained complete response and complications rates after radiofrequency ablation of very early hepatocellular carcinoma in cirrhosis: is resection still the treatment of choice? Hepatology. 2008;47(1):82-89.

2. Chakravorty N, Jaiswal S, Chakravarty D, et al. Anesthetic management of radiofrequency tumor ablation: our experience. Indian $J$ Anesth. 2006;50(2):123-127.

3. American Society of Anesthesiologists. Practice guidelines for sedation and analgesia bynonanesthesiologists. An update report by the ASA Task Force on Sedation and Analgesia by Nonanesthesiologists. Anesthesiology. 2002;96(4):1004-1017.

4. Kim YS, Lim HK, Rhim H, et al. Ablation of hepatocellular carcinoma. Best Pract Res Clin Gastroenterol. 2014;28(5):897-908.

5. Amornyotin S, Jirachaipitak S, Wangnatip S. Anesthetic management for radiofrequency ablation in patients with hepatocellular carcinoma in a developing country. J Anesth Crit Care Open Access. 2015;3(1):00086.

6. Joung KW, Choi SS, Jang DM, et al. Comparative effects of dexmedetomidine and propofol on US-guided radiofrequency ablation of hepatic neoplasm under monitored anesthesia care: a randomized controlled study. Medicine. 2015;94(32):e1349.

7. Shiina S, Tateishi R, Arano T, et al. Radiofrequency ablation for hepatocellular carcinoma: 10-year outcome and prognostic factors. $\mathrm{Am}$ J Gastroenterol. 2012;107(4):569-577.

8. Nemcek Jr AA. Complications of radiofrequency ablation of neoplasms. Semin Intervent Radiol. 2006;23(2):177-187.

9. Amornyotin S, Leelakusolvong S, Chalayonnawin W, et al. Agedependent safety analysis of propofol-based deep sedation for ERCP and EUS procedures at an Endoscopy Training Center in a developing country. Clin Exp Gastroenterol. 2012;5:123-128.

10. Amornyotin S, Kongphlay S. Anesthetic trainee-administered propofol deep sedation for small bowel enteroscopy procedure in elderly patients. J Gastroenterol Hepatol Res. 2014;3(6):1117-1129.

11. Amornyotin S, Srikureja W, Pausawasdi N, et al. Intravenous sedation for gastrointestinal endoscopy in very elderly patients of Thailand. Asian Biomedicine. 2011;5(4):485-491.

12. Gasparovic S, Rustemovic N, Opacic M, et al. Clinical analysis of propofol deep sedation for 1,104 patients undergoing gastrointestinal endoscopic procedures: a three year prospective study. World $J$ Gastroenterol. 2006;12(2):327-330.

13. Heuss LT, Inauen W. The dawning of a new sedative: propofol in gastrointestinal endoscopy. Digestion. 2004;69(1):20-26.

14. Training guideline for use of propofol in gastrointestinal endoscopy. Gastrointest Endosc. 2004;60(2):167-172. 